\title{
User adoption of the Worktivity mobile app to help reduce occupational sedentary behaviour in an office environment
}

\author{
Matias Garcia-Constantino \\ School of Computing \\ Ulster University \\ Jordanstown, UK \\ m.garcia-constantino@ulster.ac.uk
}

\author{
Suzanne M. McDonough \\ Centre for Health and Rehabilitation \\ Technologies \\ Ulster University \\ Jordanstown, UK \\ s.mcdonough@ulster.ac.uk
}

\author{
Chris Nugent \\ School of Computing \\ Ulster University \\ Jordanstown, UK \\ cd.nugent@ulster.ac.uk
}

\author{
Marie $\mathrm{H}$. Murphy \\ Sport and Exercise Science Research \\ Institute \\ Ulster University \\ Jordanstown, UK \\ mh.murphy@ulster.ac.uk
}

\author{
Aoife Stephenson \\ Sport and Exercise Science Research \\ Institute \\ Ulster University, \\ Jordanstown, UK \\ Stephenson-A@ulster.ac.uk \\ Jacqueline L. Mair \\ Sports, Exercise and Health Science \\ Research Group \\ Edinburgh Napier University \\ Edinburgh, UK \\ j.mair@napier.ac.uk
}

\begin{abstract}
In this paper we introduce the Worktivity mobile app as a potential solution to help reduce occupational sitting in an office environment. Worktivity functions by sending hourly reminders to stand up or move in addition to showing factual information related to the benefits of being active within the office environment. The Worktivity app was used over a period of 8 weeks by 37 participants aged between 18 and 65 years old from two private office worksites in Northern Ireland. Results demonstrated how users responded to the reminders over the duration of the study with mean acknowledgement rates of $66.06 \%$ and $51.57 \%$ when using the app and when using the app with a standing desk, respectively.
\end{abstract}

\section{Occupational Sedentary Behaviour - Occupational Sitting - Technology Adoption - Digital Technology}

\section{INTRODUCTION}

Occupational Sedentary Behaviour (SB) has been associated with several negative effects on human health, such as increased mortality (Van Uffelen, 2010), diabetes mellitus (Van Uffelen, 2010) and obesity (Chau, 2012). SB comprises activities in which energy expenditure is very low (e.g. sitting or lying positions) and it is usually linked to the lifestyle choice of individuals. Occupational sitting is related to sitting for extended periods of time in front of a desk. People have the choice to be active to reduce SB, however, when working in an office environment there is a tendency to be less active by sitting for prolonged periods while engaged in work tasks (Munir, 2015). Spending too much time sitting in general has been related to the following health risks: (i) Body Mass Index (BMI) linked to obesity, (ii) cancer, (iii) cardiovascular disease, (iv) diabetes mellitus, and (v) mortality (Hamilton, 2007), (Katzmarzyk, 2009), (Marshall, 2010), (Van Uffelen, 2010). Occupational sitting has also been linked to depression, anxiety and stress symptoms (Rebar, 2014). To address these associated health risks there is a need to remind and motivate desk- based workers to be more active and to spend less time sitting.

The main reason for using a mobile app as the means for reminding and motivating users to spend less time sitting is because although there are commercial devices that monitor and provide feedback on SB to the user in real time, there are no existing apps available for self monitoring that give prompts based on real time data.

The remainder of the paper is organised as follows: Section 2 describes the Worktivity app features and functionality. Section 3 describes the data collection over the time of the study. Section 4 presents some results obtained in terms of user adoption of the Worktivity app. Finally, Section 5 presents conclusions on the preliminary results and future work.

\section{THE WORKTIVITY APP}

The Worktivity app allows users to: (i) self-monitor occupational sitting SB, (ii) set personalised goals, and (iii) receive prompts, reminders and 
educational tips. The app was developed using the Xamarin cross-platform development tool. The Android platform was considered for this study.

The Worktivity app provides a simple and intuitive interface (refer to Figure 1). The main screen presents three main sections: (a) Interval (when will the recording sitting time prompts will be sent), (b) Location, and (c) Activity Log. The Activity Log section presents three buttons that provide access to: (a) Weekly feedback chart, (b) Daily goal rating, and (c) Record sitting time. The app allows users to enter their starting work time and a daily goal of how many hours they wish to be active. Based on the user's starting work time and considering a typical work day of 8 hours, prompts to record sitting time are sent hourly within the range of working hours. The idea is that users react to the prompt and record their sitting time within the last hour in the "Record sitting time" screen (Figure 1b).

The main motivational strategy used by the Worktivity app is a chart presenting the user's sitting and active time in minutes over different days (Figure 1c). Other motivational means used by the app are: (a) daily factual information about the benefits of being more active at the office sent after recording the last sitting time of a working day, (b) a daily goal rating based on how close the users are to their daily active goals, and (c) a prompt message encouraging the users to be more active if they did not achieve their hourly active time goal.

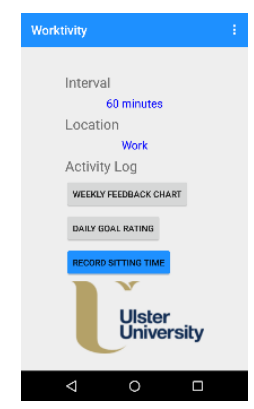

(a)

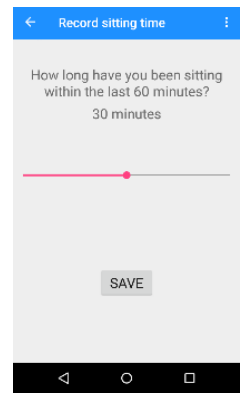

(b)

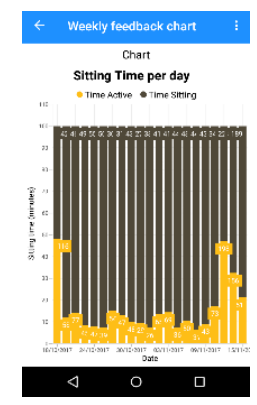

(c)
Figure 1: Screenshots of the Worktivity app showing: (a) main menu screen, (b) record sitting time screen, and (c) weekly feedback chart.

\section{INTERVENTION AND DATA COLLECTION}

This study obtained ethical approval from Ulster University's School of Sport Ethics Filter Committee. The intervention and data collection was carried out over 8 weeks and involved 37 desk-based office workers aged between 18 and 65 years old. The participants were divided into three groups: (i) using mobile app, (ii) using mobile app and sit-stand workstation desk, and (iii) control.

The daily interaction of the Worktivity app involved three main steps: (i) opening the app, (ii) reacting to the app's hourly prompts by recording sitting time, and (iii) receiving an informational tip after recording the last sitting time of the day. At any moment between the main steps it was possible for the participants to check their feedback chart and their daily goal rating when enabled.

The main issues in the data collection process related to the users were: (i) inconvenience in charging and carrying the study mobile phone in addition to their personal mobile phone, (ii) ignoring the prompts to record data, and (iii) forgetting to record data because of being busy at that time or because of a decrease in the interest after the intervention started.

\section{RESULTS}

Analysis of the results from the user adoption of the Worktivity app are divided into three groups: (i) users that used only the app, (ii) users that used the app with a sit-stand workstation desk, and (iii) all users (app, and app and desk). The collected data considered was from 42 working days: 8 weeks (40 days) and 2 test days. The Worktivity app sent prompt alerts every hour during a working day, thus a total of 336 (42 days $x 8$ working hours) prompt alerts were sent during the duration of the study to each participant.

Mean and median results were obtained for acknowledgement and response time of participants to the Worktivity app prompt alerts. In this context, the acknowledgement of a prompt alert meant that a participant reacted to a prompt alert by recording their sitting time during the previous hour. Participants that only used the app acknowledged prompt alerts more than the participants that used the app in conjunction with a standing desk (refer to Table I).

Around $30 \%$ of the time participants recorded their sitting time within a minute after a prompt alert was sent. In the case when the participants used only the app it was $34.87 \%$ and in the case when the users used the app and the standing desk it was $30.45 \%$. The mean response times were in the region of 18 minutes when the participants used only the app, and 19 minutes when they used the app in addition to the standing desk.

\section{CONCLUSIONS}

This paper has presented a study of the user adoption of Worktivity, a mobile app to help reduce occupational SB in an office environment. The user adoption results obtained were presented in terms of: (i) acknowledgement of prompt alerts sent by the app, and (ii) user response times when a prompt alert was sent. In both cases the action that confirmed the participant's acknowledgement was 
when they recorded their sitting time from the last hour. The mean acknowledgement rates were $66.06 \%$ and $51.57 \%$ in the cases of using just the app and the app in conjunction with a standing desk respectively. These results are encouraging considering that the intervention was carried out over 8 weeks and that some participants were gradually less engaged as the intervention progressed.

Future work will include adding more motivational features to the app for a better user engagement. It is planned a fine grained time series analysis regarding the participants' engagement with the Worktivity app over the 8 weeks of the intervention. It is also planned to analyse the data collected from the activPAL activity trackers and its relation to the data collected with the Worktivity app.

\section{ACKNOWLEDGMENT}

Invest Northern Ireland is acknowledged for partially supporting this project under the Competence Centre Programme Grant RD0513853 - Connected Health Innovation Centre.

\section{REFERENCES}

Chau, J. Y., van der Ploeg, H. P., Merom, D., Chey, T., and Bauman, A. E. (2012). Cross-sectional associations between occupational and leisuretime sitting, physical activity and obesity in working adults. Preventive Medicine, Vol. 54, No. 3-4, pp. 195-200, Elsevier.

Hamilton, M. T., Hamilton, D. G., and Zderic, T. W. (2007). Role of Low Energy Expenditure and Sitting in Obesity, Metabolic Syndrome, Type 2 Diabetes, and Cardiovascular Disease. Diabetes, American Diabetes Association, Vol. 56, No. 11, pp. 2655-2667.

Katzmarzyk, P. T., Church, T. S., Craig, C. L., and Bouchard, C. (2009). Sitting Time and Mortality from All Causes, Cardiovascular Disease, and Cancer. Medicine \& Science in Sports \& Exercise Journal, Vol. 41, No. 5, pp 998-1005, Lippincott Williams \& Wilkins.

Marshall, S. and Gyi, D. (2010). Evidence of Health Risks from Occupational Sitting - Where Do We Stand? American Journal of Preventive Medicine, Vol. 39, No. 4, pp. 389-391, Elsevier.

Munir, F., Houdmont, J., Clemes, S., Wilson, K., Kerr, R., and Addley, K. (2015). Work engagement and its association with occupational sitting time: results from the Stormont study. BMC Public Health Journal, BioMed Central, Vol. 15, No. 1, pp. 1-12.
Rebar, A. L., Vandelanotte, C., van Uffelen, J., Short, C., and Duncan, M. J. (2014). Associations of overall sitting time and sitting time in different contexts with depression, anxiety, and stress symptoms. Mental Health and Physical Activity Journal, Vol. 7, No. 2, pp. 105-110. Elsevier.

Van Uffelen, J. G. Z., Wong, J., Chau, J. Y., van der Ploeg, H. P., Riphagen, I., Gilson, N. D., Burton, N. W., Healy, G. N., Thorp, A. A., Clark, B. K., Gardiner, P. A., Dunstan, D. W., Bauman, A., Owen, N., and Brown, W. J. (2010). Occupational Sitting and Health Risks: A Systematic Review. American Journal of Preventive Medicine, Vol. 39, No. 4, pp. 379388, Elsevier. 